\title{
"Avoid the crush": hazards of medication administration in patients with dysphagia or a feeding tube
}

This is the first in a series of articles focusing on medication safety coedited by David $U$ of the Institute for Safe Medication Practices Canada and Edward Etchells of Sunnybrook and Women's College Health Sciences Centre.

CASE 1: A 70-year-old woman is admitted with an acute dysphagic stroke. A pureed and thickened liquid diet is started during her hospital stay. Efforts at rehabilitation are hampered by severe knee pain from chronic osteoarthritis. Her pain is initially managed with a short-acting preparation of oxycodone hydrochloride, which is then switched to an analgesicequivalent dose of the same drug in a sustained-release preparation. The next day the patient is found to be unresponsive.

CASE 2: A 75-year-old man with a percutaneous endoscopic gastrostomy tube complains of severe heartburn and undergoes endoscopy. He is found to have severe reflux esophagitis and is given omeprazole, $20 \mathrm{mg}$ twice daily, to be administered through the feeding tube. After 1 month of therapy the patient's symptoms have not resolved.

$\mathrm{B}$ oth of the patients described above experienced an adverse drug event that shared a contributing cause.

In case 1 , the patient was prescribed a tablet that she was unable to swallow whole. Because of a lack of knowledge of the characteristics of the product, the tablet was crushed for ease of administration. Oxycodone is a potent opioid, and the sustained-release formulation is designed to deliver the drug gradually over 12 hours. Crushing the tablet destroyed the drug's sustained-release properties and led to rapid absorption of the entire 12-hour dose, which resulted in sedation and respiratory depression.

In case 2, the desired benefit of the drug therapy was not obtained because the tablet was crushed. Omeprazole is formu- lated as an enteric-coated tablet to avoid inactivation of the drug by gastric acid. Crushing the tablet compromised the protective coating, which resulted in loss of efficacy.

Drugs are increasingly being marketed in an array of sophis- ticated formulations that are intended to improve the efficacy of the agent. However, the resulting availability of multiple products with similar names and a lack of caregiver understanding of the properties of the various preparations create a significant risk of adverse drug events. Little has been published about adverse events resulting from the improper use of certain dosage formulations. Lesar ${ }^{1}$ characterized prescribing errors involving or related to medication dosage forms in hospital patients. The most common error (in $70 \%$ of cases) was failure to specify the extended-release formulation when it was intended (e.g., prescribing nifedipine $60 \mathrm{mg}$ orally once daily instead of nifedipine XL $60 \mathrm{mg}$ ). These errors were identified and intercepted by pharmacists, but many had the potential for serious consequences if the short-acting formulation had been given in the dose intended for the longacting drug. Another common error (in $12 \%$ of cases) was the prescribing of extended-release formulations for administration through a feeding tube, which can also result in adverse consequences. ${ }^{1}$ The risk of errors related to dosage formulation is not limited to prescribing; errors may also occur at other stages, including the review of the medication order and drug preparation, dispensing and administration. ${ }^{1,2}$

Many patients, especially those in hospital or long-term care institutions, have swallowing disorders or require feeding tubes. These patients have trouble ingesting solid forms of drugs (e.g., tablets and capsules) orally. For some medications, liquid formulations are available for oral administsration and may serve as a suitable option, except in patients with dysphagia for liquids. However, in most situations an oral liquid formulation is not available, and opening the capsule or crushing the tablet for mixing with food or administration through a feeding tube is necessary. Although this is a reasonable option for many types of capsules and uncoated, compressed tablets, there are certain drug formulations that should not be crushed (Table 1). ${ }^{3}$ Doing so may alter the intended effect of the drug and, in some cases, may cause an adverse event.

Institutions may wish to adopt the following strategies to reduce the risk of adverse events related to improper administration of various dosage formulations:

- Development of methods for close communication between the physician, speech language pathologist (if involved in swallowing assessment), nurse and pharmacist regarding the patient's swal- 


\section{Practice}

lowing ability. For example, the speech language pathologist could write suggestions in the physician's orders regarding the patient's ability to swallow medications, and a copy of this suggestion could be sent to the pharmacy. (Sunnybrook and Women's College Health Sciences Centre has developed a communication method that is available from the author.)

- Development of pharmacy computer alerts indicating the swallowing status of individual patients to guide the pharmacist in selecting a product.
- Inclusion of instructions on the medication administration record that guide the nurse regarding appropriate administration of specific drugs (e.g., "Open capsule and mix intact beads in pureed food"; "Mix liquid medication in pureed food").

- Creation of lists of commonly used medications or drugs on the formulary that should not be crushed, with suggestions for alternative products.

- Development of a computerized physician order entry system that provides decision support for checking overly

Table 1: Drug formulations that should not be crushed ${ }^{3}$

\begin{tabular}{|c|c|c|}
\hline Type of formulation & $\begin{array}{c}\text { Common terms } \\
\text { and abbreviations }\end{array}$ & Reasons for the formulation \\
\hline Enteric-coated & $\begin{array}{l}\text { Delayed-release } \\
\text { EC: enteric-coated }\end{array}$ & $\begin{array}{l}\text { Designed to pass through the stomach } \\
\text { intact and deliver the drug for release in } \\
\text { the intestines. The formulation } \\
\text { - prevents destruction of the drug by } \\
\text { gastric acid } \\
\text { - prevents irritation of the stomach } \\
\text { - delays onset of action }\end{array}$ \\
\hline Extended-release & $\begin{array}{l}\text { CD: controlled delivery } \\
\text { CR: controlled release } \\
\text { LA: long acting } \\
\text { PA: prolonged action } \\
\text { SR: slow release } \\
\text { SR: sustained release } \\
\text { XL: extended release } \\
\text { XR: extended release }\end{array}$ & $\begin{array}{l}\text { Designed to release the drug over an } \\
\text { extended period to allow less frequent } \\
\text { administration. Formulations include } \\
\text { - multiple-layered tablets that release the } \\
\text { drug as each layer is dissolved } \\
\text { - mixed-release pellets that dissolve at } \\
\text { different intervals } \\
\text { - special matrixes that are inert but allow } \\
\text { slow release of the drug }\end{array}$ \\
\hline
\end{tabular}

high doses (e.g., if the dose is intended for an extendedrelease formulation but a regular-release product is selected) and warning against prohibited routes of administration (e.g., giving an enteric-coated preparation through a feeding tube).

With increased recognition of this problem, as well as improvements in health care provider knowledge and enhancements to medication use systems, adverse events related to sustained-release and entericcoated formulations of drugs can be avoided.

Patricia Cornish

Patient Safety Service

Department of Pharmacy

Sunnybrook and Women's College

Health Sciences Centre

Toronto, Ont.

Acknowledgement: I thank Lawrence Jackson, Department of Pharmacy, and Jane Little, Aging and Veteran's Care, Sunnybrook and Women's College Health Sciences Centre, Toronto, Ont., for their helpful comments.

\section{References}

1. Lesar TS. Prescribing errors involving medication dosage forms. $7 \mathrm{Gen}$ Intern Med 2002;17:579-87.

2. Burge FI. MS Contin: errors in prescribing, dispensing, and administering. CMA7 1986;135:96.

3. Mitchell JF. Oral dosage forms that should not be crushed: 2000 update. Hospital Pharmacy 2000;35:553-67. 